Lauro Miranda Demenech ${ }^{1,2}$

(1) https://orcid.org/0000-0002-7285-2566

Samuel C. Dumith²

Ohttps://orcid.org/0000-0002-5994-735X

Carolina Siomionki Gramajo

@ohttps://orcid.org/0000-0003-3560-5715

Maile Zanotta Ferreira'

Ohttps://orcid.org/0000-0001-7623-0724

Roberta Rodrigues Silveira

Ohttps://orcid.org/0000-0001-7328-4647

Lucas Neiva-Silva1,3

Ohttps://orcid. org/0000-0002-7526-2238

\section{Uso de club drugs entre estudantes de graduação: prevalência, características associadas e a influência dos pares}

\author{
Club drugs use among undergraduate students: prevalence, \\ associated characteristics and peer influence
}

DOI: $10.1590 / 0047-2085000000301$

\section{RESUMO}

Objetivo: Medir a prevalência do uso na vida, no último ano e no último mês de duas club drugs - o ecstasy e o LSD -, bem como as características associadas ao uso dessas substâncias, no último mês, entre estudantes de graduação de uma universidade no Sul do Brasil. Métodos: Trata-se de um estudo transversal conduzido no ano de 2015 com amostragem aleatória sistemática por conglomerados. Participaram 1.423 estudantes de graduação. Foi utilizado um modelo de análise multivariável em três níveis hierárquicos por meio da regressão de Poisson com ajuste robusto da variância. Resultados: As prevalências de uso na vida, no último ano e no último mês de club drugs foram de 12,7\%, 7,8\% e $3,8 \%$, respectivamente. Indivíduos do sexo masculino, com orientação sexual homossexual ou bissexual, mais jovens, que moravam com seus pares, solteiros, e que relataram uso no último mês de tabaco e maconha apresentaram maior probabilidade de ter feito uso no último mês de alguma club drug. Entretanto, a variável mais fortemente associada a esse desfecho foi ter algum amigo que já fez uso de alguma droga ilícita na vida $(\mathrm{RP}=19,54)$. Conclusões: $\mathrm{O}$ ambiente universitário parece ser um terreno fértil para a difusão do uso de club drugs. O fortalecimento de uma rede de apoio institucional da universidade, capaz de propor atividades de prevenção, bem como de identificar, acolher e encaminhar casos em que haja abuso e dependência dessas substâncias, pode ser uma estratégia importante para lidar com essa problemática.

\section{PALAVRAS-CHAVE}

Ecstasy, LSD, club drugs, estudantes, universidade.

\section{ABSTRACT}

Objective: To measure the prevalence of lifetime, last-year, and last-month use of two club drugs ecstasy and LSD -, as well as the characteristics associated with the last-month use of these substances among undergraduate students at a university in southern Brazil. Methods: This was a cross-sectional study conducted in 2015 with a clustered systematic sampling strategy. Overall, 1,423 undergraduate students participated. A three-level hierarchical multivariate analysis model was used through Poisson regression with robust adjustment of variance. Results: Prevalence of lifetime, last-year, and lastmonth use of club drugs were $12.7 \%, 7.8 \%$ and 3.8\%, respectively. Male participants, with homosexual or bisexual sexual orientation, younger, who lived with their peers, who were single, and who reported last-month use of tobacco and marijuana had higher probability of last-month use club drugs. However, the variable most strongly associated with this outcome was having a friend with lifetime use of illicit drugs ( $P R=19.54)$. Conclusions: University environment seems to be a fertile ground for the spread of the use of club drugs. The strengthening of the university's institutional assistance network, capable of proposing prevention activities, as well as identifying, supporting and referring cases where there is abuse and dependence on these substances can be an important strategy to deal with this problem.

\section{KEYWORDS}

Ecstasy, LSD, club drugs, students, university.
Received in: June/26/2020. Approved in: Nov/1/2020

1 Universidade Federal do Rio Grande, Centro de Estudos Sobre Risco e Saúde, Rio Grande, RS, Brasil.

2 Universidade Federal do Rio Grande, Programa de Pós-Graduação em Ciências da Saúde, Rio Grande, RS, Brasil.

3 Universidade Federal do Rio Grande, Programa de Pós-Graduação em Psicologia, Rio Grande, RS, Brasil.

Address for correspondence: Lauro Demenech. Av. Itália, km 8 - 96201-900 - Rio Grande, RS, Brasil. E-mail: lauro_demenech@hotmail.com 


\section{INTRODUÇÃO}

A história do uso de substâncias com atividade psicoativa [que atuam no sistema nervoso central (SNC) com a capacidade de modificar comportamento, humor e cognição'] se confunde com a história da humanidade. Existem evidências arqueológicas que indicam ter havido uso de cerveja em 11.000 a.C. e de maconha em 8.200 a.C. por Homo sapiens que viviam onde hoje são Israel e Japão, respectivamente?2. Contudo, é no século XX que essas substâncias começam a ser produzidas em laboratório, inicialmente com objetivos terapêuticos. Durante as décadas de 1960 e 1970, intensificam-se os estudos sobres as possibilidades de tratamento com o uso dessas substâncias. Paralelamente, começou a crescer nos Estados Unidos o movimento do uso recreativo dessas drogas, principalmente entre estudantes universitários $^{3}$. Atualmente, existem diversos tipos de drogas sintéticas, e o número de substâncias alucinógenas e estimulantes laboratorialmente manipuladas vem crescendo ${ }^{4}$. No contexto brasileiro, as drogas sintéticas mais prevalentes são o ecstasy (3,4-metilenodioximetanfetamina - MDMA) e o LSD (dietilamida do ácido lisérgico). Essas substâncias atuam primeiramente como perturbadoras do SNC, produzindo, também, efeitos estimulantes ${ }^{1,3}$.

O ecstasy é uma anfetamina simpaticomimética com uma estrutura química semelhante à da serotonina, sendo habitualmente distribuída sob a forma de pequenos comprimidos, cápsulas ou pó branco. Essa droga estimula a liberação simultânea de dopamina, serotonina e noradrenalina, agindo como estimulante e psicodélico, induzindo sentimentos de aumento de energia e prazer, principalmente por meio de estimulação tátil. Seus efeitos duram cerca de três a seis horas, e as doses são repetidas frequentemente para manter os efeitos ${ }^{5}$. O LSD, por sua vez, é um produto sintético com ação alucinógena ou psicodélica, primeiramente produzido na sua forma cristalina. Geralmente se apresenta sob forma de barras, cápsulas, tiras de gelatina, micropontos e, principalmente, em folhas de papel perfurado embebidas com a substância. Os efeitos dependem da dose administrada, da tolerância, de experiências anteriores, de aspectos psicológicos e do ambiente social ${ }^{6}$. O uso do LSD pode produzir alterações do pensamento, do humor, sensoperceptivas (principalmente visuais e auditivas), que podem gerar sensações de euforia, maior senso de conexão e compaixão consigo mesmo e com os outros, redução na ansiedade e aumento na libido?.

Novas drogas sintéticas são produzidas com grande velocidade, dificultando o entendimento de seus possíveis danos à saúde no curto, médio e longo prazo. Substâncias sintéticas alucinógenas e estimulantes parecem ser utilizadas, em sua maioria, por jovens adultos que participam do cenário das festas de músicas eletrônicas ${ }^{8}$. Apesar de existirem diversos subtipos de drogas sintéticas, o ecstasy e o LSD se consolidaram como substâncias tradicionais nesses ambientes ${ }^{9}$. Em razão dessa característica marcante do uso recreativo no contexto das festas, bares e raves, essas substâncias têm sido chamadas de club drugs ${ }^{9}$. Apesar de a prevalência de uso na vida de club drugs não ser elevada na população geral brasileira - entre $0,7 \%$ e $0,8 \%$ - ${ }^{10}$, entre frequentadores de festas a proporção de indivíduos que já experimentaram ecstasy e LSD é de $45,2 \%$ e 25,5\%, respectivamente ${ }^{11}$.

Entre as principais motivações para o uso desse grupo de drogas, usuários reportam o desejo de sentirem-se com mais energia e resistentes, para experimentar um estado de transe, ficar mais desinibidos e não pensar em problemas ${ }^{12}$. Pessoas podem acreditar que club drugs são seguras, pois muitas vezes se apresentam de forma semelhante a medicamentos prescritos ${ }^{13}$. Apesar dessas drogas poderem produzir efeitos de curto prazo desejáveis, existem riscos de complicações graves. Dentre elas, destacam-se a possibilidade de sangramento hipertensivo, desidratação, hipertermia, crises psicóticas e até coma e morte. No longo prazo, o uso crônico pode produzir sintomas depressivos, degeneração de axônios de neurônios serotoninérgicos, redução de matéria cinzenta no cérebro e diminuição na atividade do hipocampo ${ }^{7,8}$.

Existe crescente evidência da propagação do uso dessas substâncias, particularmente entre subgrupos específicos, como, por exemplo, estudantes ${ }^{14}$. Pesquisas realizadas com universitários descrevem que aproximadamente metade dos estudantes relata ter experimentado pelo menos uma droga ilícita durante a vida. A prevalência de uso na vida de ecstasy e outros alucinógenos parece ser duas até dez vezes maior que na população geral ${ }^{10,15}$ e apresenta um aumento significativo em relação às outras drogas, sendo maior entre indivíduos jovens do sexo masculino ${ }^{16,17}$.

Uma breve busca nas bases de dados apontará uma vasta quantidade de pesquisas que buscam investigar e compreender o uso de álcool, tabaco, maconha e cocaína entre universitários brasileiros. Contudo, mesmo existindo diversos relatos internacionais sobre o crescimento no uso de club drugs, especialmente entre jovens adultos, existem poucos estudos sobre esse comportamento no Brasil. Portanto, tendo em vista o exposto, o objetivo geral deste estudo foi medir a prevalência das club drugs ecstasy e LSD e identificar características individuais associadas a esse comportamento em uma amostra representativa de graduandos de uma universidade pública do Sul do Brasil.

\section{MÉTODOS}

\section{Delineamento e população-alvo}

Este estudo possui um delineamento transversal quantitativo e tem como população-alvo os estudantes de graduação da Universidade Federal do Rio Grande (FURG). Foram considerados elegíveis os estudantes com idade igual ou superior 
a 18 anos, que estudavam na modalidade presencial, regularmente matriculados no primeiro semestre de 2015. Foi utilizado como critério de exclusão ter abandonado a disciplina no momento da coleta de dados, pois a localização desses indivíduos, um a um, prejudicaria a natureza confidencial e impessoal da pesquisa.

\section{Amostragem}

Para a realização desta pesquisa, utilizou-se a técnica de amostragem sistemática por conglomerados, feita em um único estágio, a partir da relação de todas as disciplinas obtidas no sistema da universidade. Portanto, a unidade amostral desta pesquisa foram as turmas, que foram definidas como o conjunto de indivíduos matriculados em uma disciplina. Como o processo amostral foi feito a partir da listagem das turmas, foi levado em conta o efeito do delineamento amostral. Para esse cálculo, foram utilizados o tamanho do conglomerado (número médio de alunos em cada turma, estipulado em 20) $)^{15}$ e o coeficiente de correlação intraclasse (assumido como sendo 0,02) ${ }^{18}$. Assim, o valor obtido para o efeito de delineamento foi de 1,5.

Após essa etapa, foram realizados dois cálculos de tamanho amostral, um para prevalência e outro para fatores associados. No cálculo amostral descritivo, utilizou-se como prevalência estimada para o desfecho 10\%. Também se adotou uma margem de erro de dois pontos percentuais e nível de significância de 5\%. Assim, foi calculado um N de 780 indivíduos. Acrescentando 10\% para possíveis perdas e multiplicando pelo efeito de delineamento amostral esperado, obteve-se um N de 1.290 indivíduos. Para o cálculo de fatores associados, assumiram-se como parâmetros razão de proporção expostos/não expostos de 1 para 4, razão de prevalência (RP) de 1,8, poder de $80 \%$ e nível de significância de $5 \%$. Por fim, foram acrescidos ao total mais $10 \%$ para possíveis perdas e recusas, $15 \%$ para controle de fatores de confusão e 50\% para o efeito de delineamento amostral esperado. Portanto, o N final obtido foi de 1.811. Na relação obtida no sistema constavam 2.107 turmas.

Levando em conta o número médio de alunos por disciplina ser de aproximadamente $20^{15}$ e o tamanho necessário para amostra de 1.811 alunos, seriam necessárias 91 turmas. Entretanto, para garantir o êxito em atingir o tamanho amostral necessário, e considerando que um mesmo aluno poderia estar em mais de uma turma e que alguns não teriam 18 anos completos, acrescentou-se 10\% ao tamanho de amostra previamente calculado. Portanto, seriam necessárias 101 turmas para compor o processo amostral, as quais foram sistematicamente selecionadas do sistema da universidade a partir de um intervalo de seleção previamente calculado.

\section{Variáveis e instrumento}

Um questionário autoadministrado e confidencial foi utilizado. Foram avaliados os usos na vida (experimentação), no ano (últimos 12 meses) e no mês (últimos 30 dias) de LSD e ecstasy. As perguntas sobre uso das substâncias incluídas nesse estudo foram estruturadas de acordo com recomendações da Organização Mundial da Saúde (OMS) para estudos sobre o uso de drogas com estudantes ${ }^{19}$. Mais especificamente, para medir esses diferentes tipos de uso, segue-se um encadeamento de perguntas sobre experimentação ("Você já experimentou alguma vez na sua vida ecstasy?" e "Você já experimentou alguma vez na sua vida LSD?"), uso nos últimos 12 meses que antecederam a pesquisa ("Usou essa substância pelo menos uma vez nos últimos 12 meses?") e uso nos últimos 30 dias ("Usou essa substância pelo menos uma vez nos últimos 30 dias?"). Essa forma de avaliar o uso de drogas é amplamente utilizada, por exemplo, nos levantamentos nacionais sobre uso de drogas entre a população geral brasileira ${ }^{10}$ e de estudantes universitários ${ }^{15}$. Essa estratégia permite maior comparabilidade entre estudos, independentemente da população e do contexto em questão ${ }^{19}$.

A variável desfecho foi operacionalizada como o uso na vida, no ano e no mês de club drugs, que consistiu no uso de pelo menos uma dessas substância no respectivo período. Os participantes também foram questionados sobre questões socioeconômicas, demográficas e comportamentais, especificamente: sexo; orientação sexual; idade; cor da pele; renda familiar; escolaridade dos pais (tercis); migração acadêmica; situação de moradia; situação de relacionamento; uso de drogas ilícitas por familiares; uso de drogas ilícitas por amigos; prática religiosa; e uso de drogas lícitas pelo participante (uso no mês de álcool e tabaco) e uso no mês de outra droga ilícita avaliada no estudo, a maconha.

\section{Procedimentos}

Esta pesquisa foi aprovada pelo Comitê de Ética em Pesquisa da Área de Saúde (Cepas) da Universidade Federal do Rio Grande sob o número de registro 37/2015. Um estudo-piloto foi realizado na Universidade Federal de Pelotas (UFPel). Nessa ocasião, a aplicação do questionário foi cronometrada e problemas de interpretação foram avaliados e corrigidos para a versão final do instrumento. Os dados foram coletados entre abril e junho de 2015. Inicialmente, entrou-se em contato com todos os professores responsáveis pelas disciplinas selecionadas e agendaram-se o dia e o horário de aplicação do instrumento na aula. Os estudantes foram informados de que a participação era voluntária e a não participação não acarretaria danos individuais. Aqueles que concordaram em participar foram solicitados a assinar um Termo de Consentimento Livre e Esclarecido (TCLE).

Cada participante respondeu ao instrumento e depositou-o em uma urna, como estratégia para aumentar o sigilo e a confiabilidade das respostas. Cada turma foi visitada pelo menos duas vezes para minimizar as perdas do estudo. Após duas visitas, as turmas que tiveram mais de 10 perdas foram revisitadas. Indivíduos que não foram encontrados nessas 
revisitas ou que se recusaram a participar foram considerados como perdas. Os questionários preenchidos foram digitados duplamente por diferentes profissionais utilizando o software Epidata versão 3.1 .

\section{Análise de dados}

As análises estatísticas foram realizadas utilizando o software Stata IC 13.1. A análise univariada foi realizada para descrever a amostra, calculando a prevalência das variáveis independentes e do desfecho. Análises bivariadas foram conduzidas para calcular a frequência de uso de club drugs no último mês associada às variáveis de exposição. A análise multivariável foi feita por meio de regressão de Poisson com ajuste robusto para variância ${ }^{20}$. Para tanto, elaborou-se um modelo hierárquico de análise de três níveis ${ }^{21}$, no qual as variáveis foram controladas para aqueles do mesmo nível ou de níveis mais elevados. Esse modelo de regressão utiliza o teste de Wald para heterogeneidade de proporções para verificar se houve diferença significativa do desfecho entre as categorias de exposição. Para testar a associação linear entre a idade e o uso de club drugs, foi realizado o teste de Wald para tendência linear. As diferenças foram descritas em RPs, com os respectivos intervalos de confiança de 95\% (IC95\%) e valores $p$.

As variáveis foram selecionadas para o modelo final por meio do método backward. Apenas as variáveis com valor-p $\leq 0,2$ foram mantidas no modelo como estratégia de controle de confusão. As estimativas foram calculadas levando em consideração o efeito de delineamento. O nível de significância foi estabelecido em 5\% para testes bicaudais.

\section{RESULTADOS}

Foram sorteadas 101 turmas, das quais oito foram excluídas por não terem alunos matriculados. A amostra final foi composta por 93 turmas. No total, foram contabilizadas 2.459 matrículas, das quais 721 eram não elegíveis (alunos com idade inferior a 18 anos, matriculados em mais de uma turma sorteada, que haviam desistido no momento da pesquisa ou que haviam solicitado trancamento da disciplina). Assim, o estudo totalizou 1.738 indivíduos elegíveis (média de 19 alunos por turma). Portanto, a amostra final do estudo incluiu um total de 1.423 estudantes, o que representa uma taxa de resposta de $81,9 \%$ e $18,1 \%$ de perdas (15,6\% não encontrados e $2,5 \%$ de recusas).

A amostra final, conforme apresentado na tabela 1, constitui-se majoritariamente de respondentes do sexo feminino $(50,7 \%)$, heterossexuais $(87,9 \%)$ com idades entre 18 e 25 anos $(67,5 \%)$, de cor de pele branca $(78,6 \%)$ e com renda familiar de até seis salários mínimos (74,3\%). Foi observado que aproximadamente um terço da amostra migrou para estudar na universidade e que 12,8\% moravam sozinhos e 21,4\%, com pares. Além disso, 57,9\% dos participantes estavam em
Tabela 1. Descrição da amostra de estudantes de graduação da FURG de acordo com variáveis sociais , econômicas e demográficas e prevalência de uso na vida, ano e mês de ecstasy e LSD - Rio Grande, RS, Brasil, 2015

\begin{tabular}{|c|c|c|}
\hline Variável & $\mathbf{n}$ & $\%$ \\
\hline \multicolumn{3}{|l|}{$\operatorname{Sexo}(N=1.401)$} \\
\hline Feminino & 711 & 50,7 \\
\hline Masculino & 690 & 49,3 \\
\hline \multicolumn{3}{|l|}{ Orientação sexual ( $N=1.414)$} \\
\hline Heterossexual & 1.243 & 87,9 \\
\hline Homossexual/Bissexual & 171 & 12,1 \\
\hline \multicolumn{3}{|l|}{ Idade $(\mathrm{N}=1.307)$} \\
\hline 18 a 21 anos & 520 & 39,8 \\
\hline 22 a 25 anos & 362 & 27,7 \\
\hline 26 a 29 anos & 153 & 11,7 \\
\hline 30 anos ou mais & 272 & 20,8 \\
\hline \multicolumn{3}{|l|}{ Cor da pele $(\mathrm{N}=1.410)$} \\
\hline Branca & 1.109 & 78,6 \\
\hline Preta, parda ou amarela & 301 & 21,4 \\
\hline \multicolumn{3}{|l|}{ Renda familiar ( $N=1.312)$} \\
\hline Até 6 SM & 975 & 74,3 \\
\hline Maior que 6 SM & 337 & 25,7 \\
\hline \multicolumn{3}{|l|}{ Migração acadêmica (N = 1.387) } \\
\hline Cidade da universidade ou cidades vizinhas & 891 & 64,2 \\
\hline Cidade do mesmo estado & 280 & 20,2 \\
\hline Cidade de outros estados & 216 & 15,6 \\
\hline \multicolumn{3}{|l|}{ Situação de moradia ( $N=1.390)$} \\
\hline Família & 915 & 65,8 \\
\hline Sozinho & 177 & 12,8 \\
\hline Pares & 298 & 21,4 \\
\hline \multicolumn{3}{|l|}{ Situação de relacionamento $(N=1.412)$} \\
\hline Solteiro & 595 & 42,1 \\
\hline Namorando/casado & 817 & 57,9 \\
\hline \multicolumn{3}{|l|}{ Uso na vida de droga ilícita por familiar ( $N=1.399$ ) } \\
\hline Não & 1.219 & 87,1 \\
\hline $\operatorname{Sim}$ & 180 & 12,9 \\
\hline \multicolumn{3}{|l|}{ Uso na vida de droga ilícita por amigo $(N=1.408)$} \\
\hline Não & 511 & 32,3 \\
\hline Sim & 897 & 63,7 \\
\hline \multicolumn{3}{|l|}{ Prática religiosa ( $\mathrm{N}=1.414)$} \\
\hline Nunca & 563 & 39,8 \\
\hline Anualmente & 343 & 24,3 \\
\hline Mensalmente & 265 & 18,7 \\
\hline Semanal/diariamente & 243 & 17,2 \\
\hline \multicolumn{3}{|l|}{ Uso no mês de álcool ( $\mathrm{N}=1.388)$} \\
\hline Não & 408 & 29,4 \\
\hline $\operatorname{Sim}$ & 980 & 70,6 \\
\hline \multicolumn{3}{|l|}{ Uso no mês de tabaco $(\mathrm{N}=1.415)$} \\
\hline Não & 1.187 & 83,9 \\
\hline $\operatorname{Sim}$ & 228 & 16,1 \\
\hline \multicolumn{3}{|l|}{ Uso no mês de maconha $(\mathrm{N}=1.412)$} \\
\hline Não & 1.175 & 83,2 \\
\hline Sim & 237 & 16,8 \\
\hline
\end{tabular}

Notas: $\mathrm{N}$ = total de respondentes por variável; $\mathrm{n}=$ frequência absoluta por categoria; $\%$ = frequência relativa por categoria; SM = salários mínimos; FURG = Universidade Federal do Rio Grande. 
um relacionamento no momento da pesquisa, 63,7\% reportaram ter ao menos um amigo que já fez uso de drogas ilícitas e 12,9\% relataram ter familiares que já utilizaram alguma droga ilícita. As prevalências de uso no mês de álcool, tabaco e maconha foram de $70,6 \%, 16,1 \%$ e $16,8 \%$, respectivamente. A prevalência de uso na vida, no ano e no mês de club drugs foram, nesta ordem, de 12,7\%, 7,8\% e 3,8\%. Quando observadas individualmente, foi identificado que o uso de LSD e ecstasy foram estatisticamente equivalentes em todas as medidas (Tabela 2).

Na tabela 3 são apresentados os resultados das análises brutas e ajustada do uso no mês de club drugs. Nas análises brutas, apenas as variáveis cor da pele e renda não apresentaram associação com o desfecho de interesse. Após a análise ajustada, permaneceram independentemente associadas ao desfecho as seguintes variáveis: ser do sexo masculino (RP = 1,89; IC95\% 1,17-3,05); identificar-se como homossexual ou bissexual ( $\mathrm{RP}=1,99$; IC95\% 1,14-3,47); morar com pares $(\mathrm{RP}=3,05$; IC95\% 1,37-6,80); estar solteiro (RP $=2,57$; IC95\% 1,39-4,77); ter amigo que usou alguma droga ilícita na vida $(\mathrm{RP}=19,54$; IC95\% 2,60-146,84); e ter relatado uso no último mês de tabaco ( $R P=2,15$; IC95\% 1,25-3,70) e maconha ( $R P=$ 8,47; IC95\% 2,79-25,69). Houve uma associação inversa entre idade e uso de club drugs, de forma que quanto menor a idade do respondente, maior a probabilidade de uso no mês dessas substâncias (valor $p$ de tendência linear $<0,001$ ). 0 modelo final de análise apresentou um $\mathrm{R}^{2}$ ajustado de 31,6\%.

\section{DISCUSSÃO}

Esta pesquisa identificou que 12,7\% relatam já ter experimentado alguma club drug, 7,8\% as usaram pelo menos uma vez nos últimos 12 meses e 3,8\% reportaram ter feito uso no último mês. A prevalência encontrada neste estudo foi maior do que na população geral brasileira, mesmo quando comparado com as faixas etárias mais novas ${ }^{10}$, bem como foi superior aos resultados encontrados no levantamento nacional sobre o uso de drogas entre universitários ${ }^{15}$. Entretanto, essas proporções encontradas em nosso estudo foram semelhantes a estudo conduzido com universitários estadunidenses ${ }^{22}$. É possível que a alta frequência de club drugs tenha ocorrido por causa das características da população universitária. Esse grupo é formado majoritariamente por adultos jovens, que vivem em uma cultura na qual frequentar festas é um hábito recorrente. Nesse contexto, as club drugs, que são drogas associadas ao ambiente de festas ${ }^{8,12}$, podem ter maior circulação, aumentando o número de usuários.

Foi observado que indivíduos do sexo masculino, mais jovens e participantes homossexuais ou bissexuais tiveram maior probabilidade de uso de club drugs, perfil semelhante ao encontrado em outras investigações com essa temáti$\mathrm{ca}^{11,12,14,23}$. Estudos indicam que homens apresentam maior percepção de facilidade no acesso a drogas ilícitas e menor percepção de risco no uso dessas substâncias, quando comparados às mulhere ${ }^{24,25}$, o que pode ser uma explicação plausível para o fenômeno observado em nossa pesquisa. Outra possibilidade é que indivíduos do sexo masculino tendem a expor-se a maiores números de situações de risco do que mulheres, um possível reflexo da masculinidade tóxica socialmente estabelecidaa, ${ }^{26,27}$. Indivíduos jovens são mais predispostos à busca por novas sensações, o que pode ter contribuído para os resultados encontrados. Além disso, é na juventude que se desenvolvem a identidade e, consequentemente, a busca pela aceitação dos pares ${ }^{28,29}$. Em relação à orientação sexual, existem evidências de que homens e muIheres homossexuais e bissexuais possuem maior probabilidade de usar drogas lícitas e ilícitas, incluindo club drugs, do que seus pares heterossexuais ${ }^{14,23,30}$. Esse resultado é importante, uma vez que o uso de club drugs entre esse subgrupo populacional tem sido associado ao aumento no número de infecções sexualmente transmissíveis, tornando-se um importante fator de risco para desfechos negativos na saúde física e mental desses indivíduos ${ }^{14,31}$.

Indivíduos solteiros apresentaram maior prevalência de uso de club drugs no último mês. O contexto universitário, principalmente a nível de graduação, apresenta uma cultura de intenso envolvimento com festas, as quais apresentam maior disponibilidade e incentivo de uso de substâncias psicoativas. Pessoas solteiras constituem a maior proporção de frequentadores de festas, ambientes voltados para a diversão e o prazer. O fator de grupo, combinado com o efeito desejado do entretenimento, é um elemento que incentiva o uso de drogas entre jovens adultos ${ }^{28}$. Além disso, existem evidências de que a diversão é uma das principais motivações para o uso de drogas sintéticas ${ }^{12}$. Ainda, festas universitárias são, em geral,

Tabela 2. Uso na vida, no ano e no mês de club drugs entre estudantes de graduação da FURG ( $N=1.423)$ - Rio Grande, RS, Brasil, 2015

\begin{tabular}{lccc}
\hline & $\begin{array}{c}\text { Uso na vida } \\
\text { \% (IC95\%) }\end{array}$ & $\begin{array}{c}\text { Uso no ano } \\
\text { \% (IC95\%) }\end{array}$ & $\begin{array}{c}\text { Uso no mês } \\
\text { (IC95\%) }\end{array}$ \\
\hline Club drugs & $12,7(10,9-14,4)$ & $7,8(6,4-9,2)$ & $3,8(2,8-4,8)$ \\
Tipos* & & & $2,0(1,3-2,7)$ \\
$\quad$ Ecstasy & $9,0(7,5-10,5)$ & $4,6(3,5-5,7)$ & $2,8(1,9-3,7)$ \\
LSD & $9,8(8,3-11,3)$ & $5,9(4,7-7,2)$ & \\
\hline
\end{tabular}

Notas: \% = prevalência; IC95\% = intervalo de confiança de 95\%; * respostas múltiplas; FURG = Universidade Federal do Rio Grande. 
Tabela 3. Razões de prevalência brutas e ajustada para associações entre uso no mês de club drugs e variáveis independentes. Análise multivariável conduzida por meio da regressão de Poisson com ajuste robusto da variância, considerando modelo hierárquico de três níveis e efeito de delineamento Amostra de estudantes de graduação da FURG ( $N=1.423)$ - Rio Grande, RS, Brasil, 2015

\begin{tabular}{|c|c|c|c|c|}
\hline \multirow{2}{*}{ Nível } & \multirow{2}{*}{ Variável } & \multirow{2}{*}{$\%$} & \multirow{2}{*}{$\begin{array}{c}\text { Bruta } \\
\text { RP (IC95\%) }\end{array}$} & \multirow{2}{*}{$\begin{array}{c}\text { Ajustada } \\
\text { RP (IC95\%) }\end{array}$} \\
\hline & & & & \\
\hline \multirow[t]{17}{*}{$1^{\circ}$} & Sexo & & $p=0,006$ & $p=0,010$ \\
\hline & Feminino & 2,6 & 1 & 1 \\
\hline & Masculino & 5,1 & 1,99 (1,22-3,23) & $1,89(1,17-3,05)$ \\
\hline & Orientação sexual & & $p=0,005$ & $p=0,016$ \\
\hline & Heterossexual & 3,3 & 1 & 1 \\
\hline & Homossexual/bissexual & 7,7 & $2,28(1,29-4,05)$ & $1,99(1,14-3,47)$ \\
\hline & Idade & & $p<0,001^{*}$ & $p=0,001^{\star}$ \\
\hline & 18 a 21 anos & 5,3 & $6,99(1,55-31,46)$ & $6,70(1,48-30,31)$ \\
\hline & 22 a 25 anos & 4,5 & $6,00(1,38-26,09)$ & $5,24(1,20-22,91)$ \\
\hline & 26 a 29 anos & 2,0 & $2,66(0,45-15,63$ & $2,33(0,38-14,21)$ \\
\hline & 30 anos ou mais & 0,8 & 1 & 1 \\
\hline & Cor da pele & & $p=0,764$ & $p=0,509$ \\
\hline & Branca & 3,7 & 1 & 1 \\
\hline & Preta, parda ou amarela & 4,1 & $1,09(061-1,98)$ & $1,22(0,67-2,22)$ \\
\hline & Renda familiar & & $p=0,757$ & $p=0,883$ \\
\hline & Até 6 SM & 3,9 & 1 & 1 \\
\hline & Maior que 6 SM & 3,6 & $0,91(0,48-1,71)$ & $0,95(0,45-1,92)$ \\
\hline \multirow[t]{17}{*}{$2^{0}$} & Migração acadêmica & & $p=0,007$ & $p=0,367$ \\
\hline & Cidade da universidade ou cidades vizinhas & 2,9 & 1 & 1 \\
\hline & Cidade do mesmo estado & 4,0 & $1,36(0,69-2,68)$ & $0,54(0,18-1,68)$ \\
\hline & Cidade de outros estados & 7,9 & $2,68(1,46-4,92)$ & $0,90(0,32-2,55)$ \\
\hline & Situação de moradia & & $p<0,001$ & $p=0,026$ \\
\hline & Família & 2,1 & 1 & 1 \\
\hline & Sozinho & 6,3 & $3,00(1,56-5,77)$ & $2,21(0,97-5,07)$ \\
\hline & Pares & 7,7 & $3,62(1,89-6,93)$ & $3,05(1,37-6,80)$ \\
\hline & Situação de relacionamento & & $p<0,001$ & $p=0,003$ \\
\hline & Solteiro & 6,1 & $2,90(1,75-4,82)$ & $2,57(1,39-4,77)$ \\
\hline & Namorando/casado & 2,1 & 1 & 1 \\
\hline & Uso na vida de droga ilícita por familiar & & $p=0,020$ & $p=0,459$ \\
\hline & Não & 3,5 & 1 & 1 \\
\hline & Sim & 6,7 & $1,89(1,11-3,24)$ & $1,31(0,64-2,70)$ \\
\hline & Uso na vida de droga ilícita por amigo & & $p=0,001$ & $p=0,004$ \\
\hline & Não & 0,2 & 1 & 1 \\
\hline & Sim & 6,0 & $30,39(4,00-230-46)$ & $19,54(2,60-146,84)$ \\
\hline \multirow[t]{14}{*}{$3^{\circ}$} & Prática religiosa & & $p=0,032$ & $p=0,785$ \\
\hline & Nunca & 6,1 & 1 & 1 \\
\hline & Anualmente & 3,8 & $0,63(0,31-1,29)$ & $0,94(0,43-2,06)$ \\
\hline & Mensalmente & 2,3 & $0,38(0,13-1,08)$ & $0,71(0,24-2,13)$ \\
\hline & Semanal/diariamente & 0,4 & $0,07(0,01-0,54)$ & $0,38(0,05-3,03)$ \\
\hline & Uso no mês de álcool & & $p=0,003$ & $p=0,311$ \\
\hline & Não & 0,3 & 1 & 1 \\
\hline & Sim & 5,2 & $20,89(2,87-152,28)$ & $2,91(0,36-23,34)$ \\
\hline & Uso no mês de tabaco & & $p<0,001$ & $p=0,006$ \\
\hline & Não & 2,3 & 1 & 1 \\
\hline & Sim & 12,1 & $5,20(3,13-8,64)$ & $2,15(1,25-3,70)$ \\
\hline & Uso no mês de maconha & & $p<0,001$ & $p<0,001$ \\
\hline & Não & 0,7 & 1 & 1 \\
\hline & $\operatorname{Sim}$ & 19,5 & $28,42(11,69-69,12)$ & $8,47(2,79-25,69)$ \\
\hline
\end{tabular}

Notas: \% = prevalência do desfecho por cada categoria; RP = razão de prevalência; IC95\% = intervalo de confiança de 95\%; * Teste de Wald para tendência linear; SM = salários mínimos; FURG = Universidade Federal do Rio Grande. 
marcadas pelo consumo excessivo de álcool. Entretanto, o uso de club drugs não esteve associado ao consumo de álcool. Esse resultado pode ser um indicativo de um contexto específico do uso dessas substâncias: as festas de música eletrônica ou raves ${ }^{31}$. Nesses ambientes é comum maior consumo de água (em detrimento de outras bebidas, como as alcoólicas), por causa dos efeitos fisiológicos e da possível reação de hipertermia que o uso dessas substâncias pode causar.

Participantes que relataram uso no último mês de tabaco e maconha tiveram maior probabilidade de ter reportado uso de club drugs. Estudantes universitários possuem maior percepção de risco do uso de tabaco e menor percepção de risco do uso de drogas ilícitas, quando comparados à população geral de jovens adultos ${ }^{25}$. Entre universitários que já fumaram em algum ponto de suas vidas, a percepção de risco do uso ocasional de drogas ilícitas parece ser baixa, possivelmente porque, na interpretação dos alunos, os possíveis riscos de longo prazo à saúde impostos por essas substâncias não serão muito piores do que aqueles aos quais eles já se expuseram ${ }^{25}$. Dessa forma, é possível que o mesmo fenômeno tenha ocorrido em nossa amostra. Quanto à maconha, outras investigações também encontraram resultados semeIhantes ${ }^{22,31,32}$. Análises temporais sobre o uso de substâncias entre universitários estadunidenses indicam que a percepção de risco das drogas ilícitas tem permanecido estável, exceto do LSD e da maconha, que diminuiu. Além disso, enquanto para a maioria das substâncias ilícitas a percepção de facilidade de acesso diminuiu, para essas duas substâncias ela permaneceu estável ${ }^{33}$. Levando em consideração que pessoas que percebem como baixo o risco de uso de uma substância apresentam maior probabilidade de usá-las ${ }^{9}$, é possível que indivíduos que usam uma substância perturbadora do SNC possam perceber outras também como de baixo risco, aumentando a probabilidade de uso de ambas.

A hipótese central deste trabalho é a influência dos pares no uso dessas substâncias. Isso já foi destacado quando foram discutidos os dados sobre idade, estado civil e uso de tabaco e maconha. Entretanto, outras duas variáveis parecem dar ainda mais consistência a essa hipótese. Primeiramente, foi identificado que indivíduos que moram com os pares tiveram probabilidade três vezes maior de ter usado club drugs no último mês, quando comparados com aqueles que moravam com familiares. Em segundo lugar, participantes que reportaram ter algum amigo que já usou alguma droga ilícita apresentaram a maior medida de efeito deste estudo, uma RP de 19,54. Observando esses dois dados de forma combinada, eles podem nos indicar dois importantes fenômenos: 1) indivíduos que moram com os pares tendem a ter menor contato com o monitoramento familiar ${ }^{34}$ e, paralelamente, podem experimentar aumento nas oportunidades e ofertas para o uso dessas substâncias, bem como uma redução em suas capacidades de recusá-las ${ }^{35,36}$; 2) tendo amigos que usam ou já usaram alguma substância ilícita, os universitários podem apresentar um aumento ainda maior no número de oportunidades para usá-las ${ }^{35}$. Associado a isso, a influência dos amigos ocorre na medida em que os jovens buscam adotar o comportamento do grupo em que estão inseridos, buscando status e sentimento de pertencimento, motivados pela busca de diversão, prazer e redução da ansiedade e do estresse ${ }^{37}$.

É importante destacar algumas limitações e fortalezas deste estudo. Primeiro, por ser um estudo transversal, não é possível estabelecer temporalidade. Portanto, as associações com variáveis comportamentais são suscetíveis ao viés de causalidade reversa. Segundo, a prevalência do uso de club drugs pode ter sido subestimada, em razão da ocorrência de respostas socialmente aceitas e pela exclusão de indivíduos que já haviam abandonado ou formalmente desistido do curso. Terceiro, comorbidades psiquiátricas não foram avaliadas, as quais podem se constituir como possíveis fontes de confusão nas associações investigadas. Quadros de abuso ou dependência de drogas podem produzir grande sofrimento psicológico (e vice-versa) ${ }^{38}$. Contudo, revisões sistemáticas e metanálises nacionais ${ }^{39,40}$ e internacionais ${ }^{41-43}$ sobre saúde mental e uso de drogas entre estudantes universitários não têm identificado associação significativa entre uso de substâncias e sofrimento psicológico. É possível que isso se deva ao fato de serem, em geral, estudos sobre uso recreativo de drogas, incluindo um grupo grande de indivíduos que não desenvolveram transtornos de uso de substâncias. Quanto aos pontos fortes, destaca-se que esta investigação contou com uma amostra expressiva, representativa e com uma taxa de resposta adequada, que garantem a validade interna deste estudo. Considerando o diminuto número de pesquisas que avaliam o uso de club drugs entre universitários brasileiros, este artigo possui caráter inédito. Por fim, o uso de estratégias de análise multivariável auxiliou na obtenção de resultados robustos e precisos, que permitem a utilização das conclusões apresentadas pelos profissionais que atuam diretamente com essa população.

\section{CONCLUSÕES}

Dentre os resultados obtidos, destacam-se as altas prevalências encontradas, quando comparadas com a população geral e de universitários brasileiros, e os fatores associados: ser do sexo masculino, ter orientação homossexual ou bissexual, ser mais jovem, estar solteiro, morar com os pares, ter amigo que já fez uso de droga ilícita e ter feito uso no último mês de tabaco e maconha. De forma geral, o contexto universitário parece ser terreno fértil para a difusão do uso de club drugs, em razão da extensa rede de pessoas que já fez uso de drogas ilícitas, por ser um ambiente marcado pela cultura 
das festas (incluindo festas eletrônicas e raves) e pela grande proporção de indivíduos jovens que moram afastados do monitoramento familiar.

Drogas sintéticas, como o ecstasy e o LSD, podem causar diversos impactos negativos no curto e longo prazo. Portanto, é importante que as universidades assumam uma posição de responsabilidade e promovam ações voltadas para a conscientização sobre o uso dessas drogas, as quais possam ser direcionadas paras os grupos de risco identificados. Intervenções como palestras, grupos de apoio, canais de informação e grupos de estudo sobre o tema podem ser medidas importantes a serem tomadas. Por fim, sugere-se o fortalecimento de serviços de assistência estudantil capazes de identificar e acolher casos de uso, abuso e/ou dependência dessas (e de outras) substâncias e de referenciá-los à rede de atenção psicossocial, quando necessário.

\section{CONTRIBUIÇÕES INDIVIDUAIS} Lauro Miranda Demenech, Samuel C. Dumith e Lucas
Neiva-Silva - Contribuíram na concepção, delineamento e condução do estudo, na análise e interpretação dos dados e na escrita e revisão técnico-científica do manuscrito.

\section{Carolina Siomionki Gramajo, Maile Zanotta Ferreira e} Roberta Rodrigues Silveira - Contribuíram na análise e interpretação dos dados e na escrita do manuscrito.

Todos os autores aprovaram a versão final do artigo, sendo totalmente responsáveis pelo seu conteúdo científico.

\section{CONFLITO DE INTERESSES}

Os autores não possuem conflito de interesses a declarar.

\section{AGRADECIMENTOS}

O primeiro autor recebeu uma bolsa de estudos da Capes (Coordenação de Aperfeiçoamento de Pessoal de Nível Superior) para conduzir este estudo. O segundo autor é bolsista de produtividade em pesquisa do CNPq (Conselho Nacional de Desenvolvimento Científico e Tecnológico).

\section{REFERÊNCIAS}

1. Galduróz JCF. Abuso e dependência de drogas. Adolescência uso e abuso de drogas: uma visão integrativa. São Paulo: Fap Unifesp; 2011.

2. Samorini $G$. The oldest archeological data evidencing the relationship of Homo sapiens with psychoactive plants: A worldwide overview. J Psychedelic Stud. 2019:1-18.

3. Centro Brasileiro de Informações sobre Drogas Psicotrópicas. Livreto informativo sobre drogas psicotrópicas: leitura recomendada para alunos a partir do 70 ano do ensino fundamental. $5^{\text {a }}$ ed. Brasília, DF: Centro Brasileiro de Informações sobre Drogas Psicotrópicas; 2011. 64p

4. Chavant F, Boucher A, Le Boisselier R, Deheul S, Debruyne D. New synthetic drugs in addictovigilance. Therapie. 2015;70(2):167-89.

5. Gudelsky GA, Yamamoto BK. Actions of 3,4-methylenedioxymethamphetamine (MDMA) on cerebral dopaminergic, serotonergic and cholinergic neurons. Pharmacol Biochem Behav. 2008;90(2):198-207.

6. Guerreiro DF, Carmo AL, Silva JA, Navarro R, Góis C. Club drugs: Um novo perfil de abuso de substâncias em adolescentes e jovens adultos. Acta Med Port. 2011;24(5):739-56.

7. Betzler F, Viohl L, Romanczuk-Seiferth N. Decision-making in chronic ecstasy users: a systematic review. Eur J Neurosci. 2017;45(1):34-44.

8. Hassen GW, Ghobadi F, Kalantari H. Synthetic drugs: a new trend and the hidden danger. Am J Emerg Med. 2013;31(9):1413-5.

9. Leung KS, Cottler LB. Ecstasy and other club drugs: a review of recent epidemiologic studies. Curr Opin Psychiatry. 2008;21(3):234-41.

10. Bastos FIPM, Vasconcellos MTL, De Boni RB, Reis NB, Coutinho CFS. III Levantamento Nacional sobre 0 uso de drogas pela população brasileira. Rio de Janeiro: Fundação Oswaldo Cruz; 2017.

11. Kelly BC, Parsons JT, Wells BE. Prevalence and predictors of club drug use among clubgoing young adults in New York city. J Urban Health. 2006;83(5):884-95.

12. Biolcati R, Mancini G. Club drugs and rave parties: A pilot study on synthetic drug consumption styles in a sample of young Italian ravers. Open Public Health J. 2018;11:474-84.

13. American Academy of Family Physicians. Club drugs: What you should know. Am Fam Physician. 2018;98(2):1-2.

14. Sullivan AK. Club drugs: what's happening? Sex Transm Infect. 2015;91(6):388.

15. Andrade AG, Duarte PCAV, Oliveira LG. I Levantamento Nacional Sobre o Uso de Álcool, Tabaco e Outras Drogas Entre Universitários das 27 Capitais Brasileiras. São Paulo: Secretaria Nacional de Políticas sobre Drogas (Senad); 2010

16. Andrade AG, Duarte PCAV, Barroso LP, Nishimura R, Alberghini DG, Oliveira LG. Use of alcohol and other drugs among Brazilian college students: effects of gender and age. Braz J Psiquiatr. 2012;34:294-305.

17. Wagner GA, Oliveira LG, Barroso LP, Nishimura R, Ishihara LM, Stempliuk VA, et al. Drug use in college students: a 13-year trend. Rev Saúde Pública. 2012;46:497-504.

18. Silva NN. Amostragem probabilística: um curso introdutório. $2^{a}$ ed. São Paulo: EDUSP; 2001.

19. World Health Organization. A methodology for student drug-use surveys. Geneva: WHO; 1980.

20. Barros AJD, Hirakata VN. Alternatives for logistic regression in cross-sectional studies: an empirical comparison of models that directly estimate the prevalence ratio. BMC Med Res Methodol. 2003;3(21)

21. Victora CG, Huttly SR, Fuchs SC, Olinto MT. The role of conceptual frameworks in epidemiological analysis: a hierarchical approach. Int J Epidemiol. 1997;26(1):224-7.

22. Wish ED, Fitzelle DB, O'Grady KE, Hsu MH, Arria AM. Evidence for significant polydrug use among ecstasy-using college students. J Am Coll Health. 2006;55(2):99-104.

23. Fazio A, Joe-Laidler K, Moloney M, Hunt G. Gender, sexuality, and ethnicity as factors of club drug use among Asian Americans. J Drug Issues. 2010;40(2):405-32.

24. Hynes M, Demarco M, Araneda JC, Cumsille F. Prevalence of Marijuana Use among University Students in Bolivia, Colombia, Ecuador, and Peru. Int J Environ Res Public Health. 2015;12(5):5233-40.

25. Bejarano J, Ahumada G, Sánchez G, Cadenas N, Marco M, Hynes M, et al. Perception of risk and drug use: An exploratory analysis of explanatory factors in six Latin American countries. Int J Alcohol Drug Res. 2011;1(1):9-17.

26. Schraiber LB, Gomes R, Couto MT. Homens e saúde na pauta da Saúde Coletiva. Ciênc Saúde Coletiva. 2005;10:7-17

27. Schwarz E. Reflexões sobre gênero e a Política Nacional de Atenção Integral à Saúde do Homem. Ciênc Saúde Coletiva. 2012;17:2581-3.

28. Andrews JA, Tildesley E, Hops H, Li F. The influence of peers on young adult substance use. Health Psychol. 2002;21(4):349-57. 
29. Papalia DE, Feldman RD. Desenvolvimento Humano. 12a ed. Porto Alegre: Artmed; 2013

30. Hahm HC, Wong FY, Huang ZJ, Ozonoff A, Lee J. Substance use among Asian Americans and Pacific Islanders sexual minority adolescents: findings from the National Longitudinal Study of Adolescent Health. J Adolesc Health. 2008;42(3):275-83.

31. Maxwell JC. Party drugs: properties, prevalence, patterns, and problems. Subst Use Misuse. 2005:40(9-10):1203-40.

32. Verduin ML, Payne RA, McRae AL, Back SE, Simpson SA, Sarang RY, et al. Assessment of club drug use in a treatment-seeking sample of individuals with marijuana dependence. Am J Addict. 2007;16(6):484-7.

33. Lipari $R$, Jean-Francois B. Trends in perception of risk and availability of substance use among full-time college students. The CBHSQ Report. Rockville (MD): Substance Abuse and Mental Health Services Administration (US); 2016.

34. Martins SS, Storr CL, Alexandre PK, Chilcoat HD. Adolescent ecstasy and other drug use in the National Survey of Parents and Youth: The role of sensation-seeking, parental monitoring and peer's drug use. Addict Behav. 2008;33(7):919-33.

35. Pinchevsky GM, Arria AM, Caldeira KM, Garnier-Dykstra LM, Vincent KB, O'Grady KE. Marijuana exposure opportunity and initiation during college: Parent and Peer Influences. Prev Sci. 2012;13(1):18

36. Kassa A, Taddesse F, Yilma A. Prevalence and factors determining psychoactive substance (PAS) use among Hawassa University (HU) undergraduate students, Hawassa Ethiopia. BMC Public Health. 2014;14
37. Santos DDM, Guimarães MM, Bodevan EC, Rocha RL, Pinheiro MLP. Use of psychoactive substance among university students. SMAD. 2019;15(3):1-9.

38. National Institute on Drug Abuse. Part 1: The connection between substance use disorders and mental illness. Disponivel em: https://www.drugabuse.gov/publications/researchreports/common-comorbidities-substance-use-disorders/part-1-connection-betweensubstance-use-disorders-mental-illness. Acesso em: 4 out. 2020

39. Candido FJ, Souza R, Stumpf MA, Fernandes LG, Veiga R, Santin M, et al. The use of drugs and medical students: a literature review. Rev Assoc Med Bras. 2018;64(5):462-8.

40. Pacheco JP, Giacomin HT, Tam WW, Ribeiro TB, Arab C, Bezerra IM, et al. Mental health problems among medical students in Brazil: a systematic review and meta-analysis. Braz J Psiquiatr. 2017;39(4):369-77.

41. Ibrahim AK, Kelly SJ, Adams (E, Glazebrook C. A systematic review of studies of depression prevalence in university students. J Psychiatr Res. 2013;47(3):391-400.

42. Lei XY, Xiao LM, Liu YN, Li YM. Prevalence of Depression among Chinese University Students: A Meta-Analysis. PLoS One. 2016;11(4):e0153454.

43. Li ZZ, Li YM, Lei XY, Zhang D, Liu L, Tang SY, et al. Prevalence of suicidal ideation in Chinese college students: a meta-analysis. PLoS One. 2014;9(10):e104368. 